\title{
ULTRASHORT ELECTRON BUNCH LENGTH MEASUREMENTS AT DUVFEL
}

\author{
W.S. Graves, G.L. Carr, L.F. DiMauro, A. Doyuran, R. Heese, E.D. Johnson, C. Neuman, G. \\ Rakowsky, J. Rose, J. Rudati, T. Shaftan, B. Sheehy, J. Skaritka, L.-H. Yu \\ NSLS, Brookhaven National Laboratory, Upton, NY 11973, USA \\ D. H. Dowell, Boeing Physical Sciences Research Center, Seattle, WA 9812
}

\section{Abstract}

The DUVFEL electron linac is designed to produce sub-picosecond, high brightness electron bunches for driving a short wavelength FEL. Four experiments have been commissioned to address the challenge of accurately measuring bunch lengths on this timescale. In the frequency domain, a short 12 period undulator is used to produce both off-axis coherent emission and on-axis incoherent single-shot spectra. The total coherent infrared power scales inversely with bunch length and the spectral cutoff is an indication of bunch length. The density of power spikes in the single-shot visible spectrum may also be used to estimate bunch length. In the time domain, the linac accelerating sections and a bending magnet are used to implement the RF-zero phasing method, and a subpicosecond streak camera is also installed. Beam measurements and comparisons of these methods are reported.

\section{RF ZERO PHASING}

The RF zero phasing method [1] uses the accelerator itself as a relativistic streak camera. A large timecorrelated energy spread (linear chirp) is induced on the beam by setting the phase of one or more linac sections to

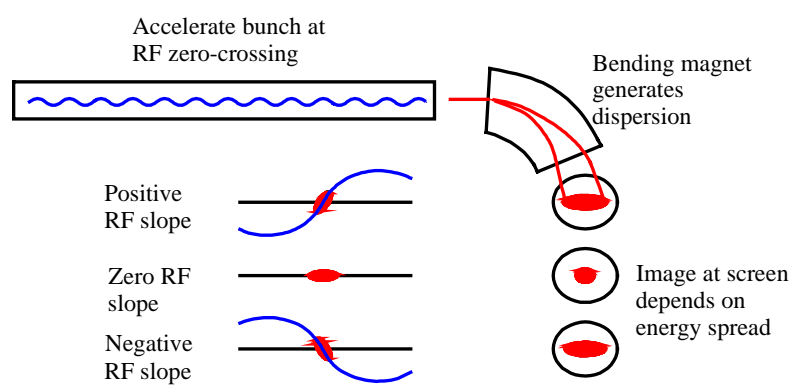

Figure 1: RF zero phasing schematic.

zero (i.e. nonaccelerating) phase. The beam is then dispersed by a dipole magnet and intercepted by a scintillating screen. The different time slices of the beam travel different trajectories depending on their energy, and thus intercept different positions on the scintillator (Fig.'s 1 and 2). Both zero crossings of the RF waveform are used so that any incoming time-energy correlations become apparent as an asymmetry in beam sizes for each of the two RF slopes. With the RF off the beam size is determined by uncorrelated energy spread and the lattice beta function. The RMS time resolution is given by

\section{Energy $=75 \mathrm{MeV}$, Charge $=20 \mathrm{pC}$, Tank 4 energy gain $=11 \mathrm{MeV}$}

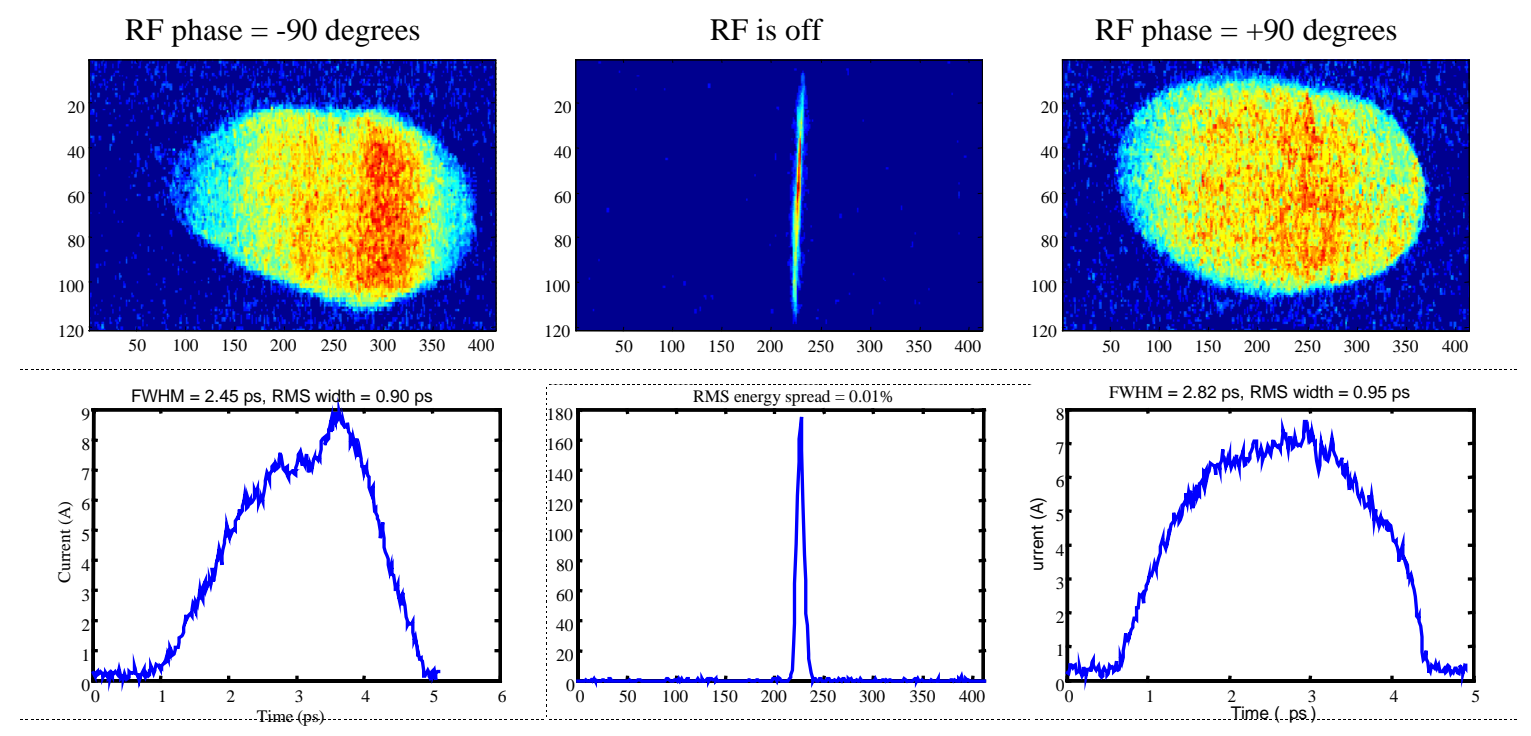

Figure 2: Upper plots are beam images on RF zero phasing scintillator. Lower are time projections 

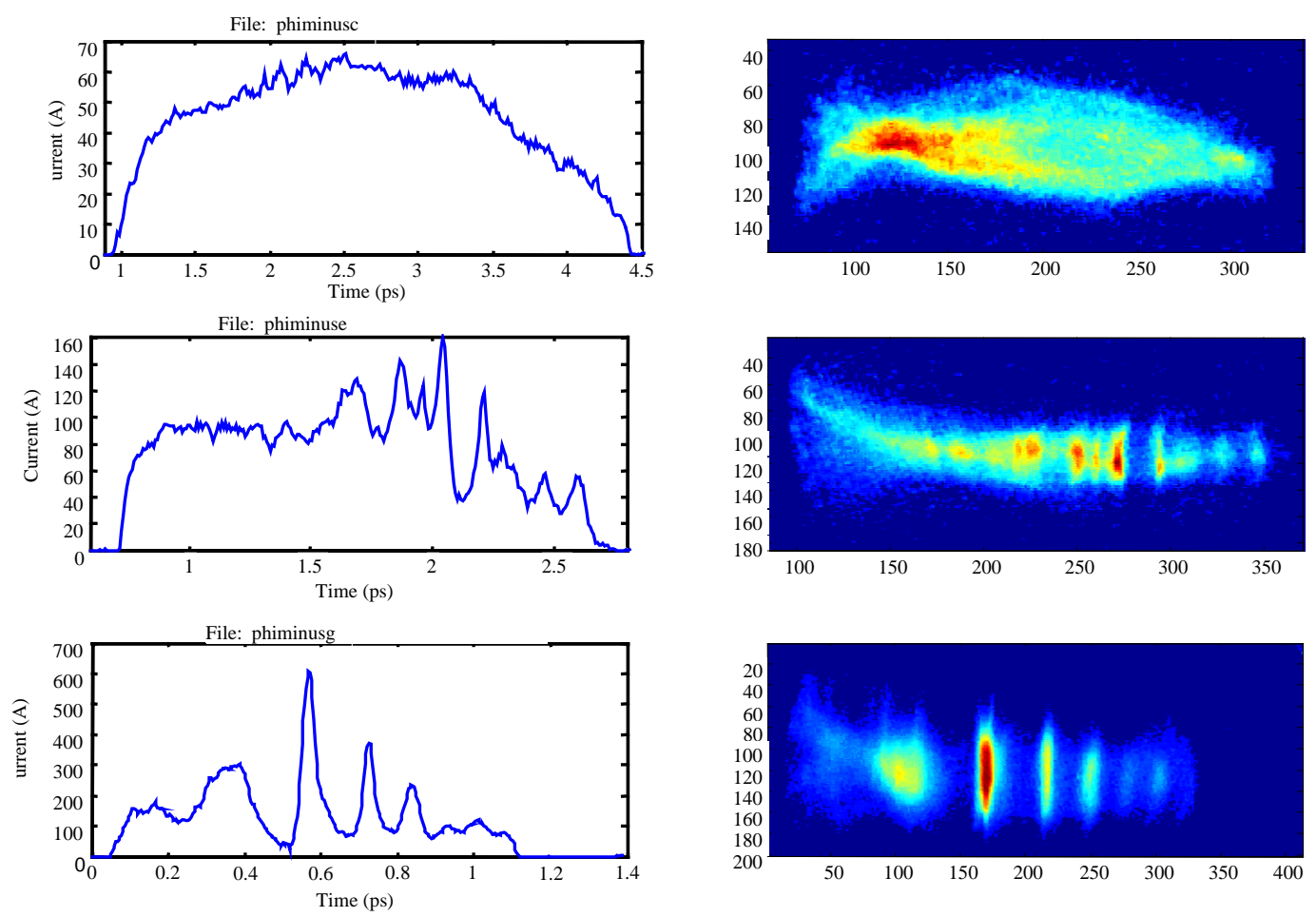

Figure 3: TOP: no compression. MIDDLE: mild compression (microbunching begins to appear). Bottom: strong compression (microbunching apparent). $\mathrm{Q}=250 \mathrm{pC}$. Note change in time scales.

$$
\Delta t=\frac{E_{0} \sigma_{x}}{\eta E_{r f} k_{r f} c}
$$

where $E_{0}=75 \mathrm{MeV}$ is the beam energy, $\sigma_{x}=120 \mathrm{um}$ is the measured beam size with RF off, $\eta=1.1 \mathrm{~m}$ is the dispersion at the scintillator screen, $E_{r f}=60 \mathrm{MV}$ is the maximum voltage in a single zero-phasing section, $k_{r f}=$ $60 \mathrm{~m}^{-1}$ is the RF wavenumber, and $c$ is the speed of light. The resolution is then $\Delta t=8 \mathrm{fs}$ at full voltage. Generally, the rf-zero phasing voltage is set at less than the maximum value so that the entire beam pulse is imaged on the $15 \mathrm{~mm}$ diameter YAG:Ce scintillator. In this case the time resolution is the ratio of beam size with RF off to that with RF on, or $120 \mathrm{um} / 15 \mathrm{~mm}=0.8 \%$. The remarkable time resolution at the DUVFEL is due to 3 factors: the linac is relatively low energy, a large energy chirp is possible after the chicane, and the 70 degree dipole produces over $1 \mathrm{~m}$ of dispersion.

\section{MICROBUNCHING OBSERVATIONS}

During compression studies, microbunching within a single electron bunch often becomes visible near maximum compression (Fig. 3). The peaks in the time distribution of this example are just 50 fs FWHM wide, and the spacing between peaks is $140 \mathrm{fs}$. These time domain measurements are confirmed by measured coherent radiation spectra [2].

The source of the microbunching is apparently variations in the laser time profile. These variations are at longer timescales of about $500 \mathrm{fs}$. However, they are not generally visible in the uncompressed electron pulse at high charge. Space charge near the cathode smoothes out local density variations when the charge is high. Although the time profile is then smooth, local energy modulations have been introduced that modify the pulse
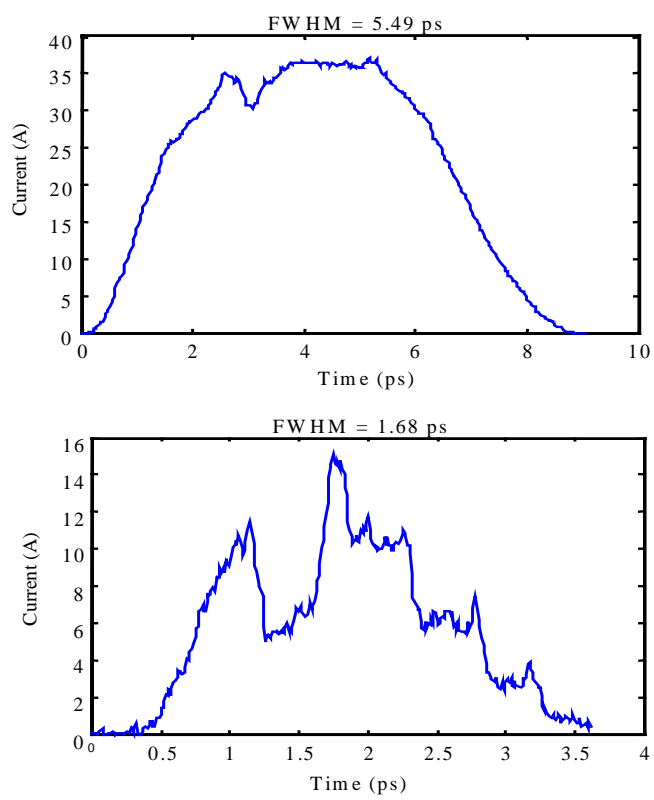

Figure 4: TOP: $300 \mathrm{pC}$ uncompressed time profile. BOTTOM: $25 \mathrm{pC}$ uncompressed time profile under same conditions. Note pulse stretching and smoothing at high charge. 
shape following compression. The nominally linear energy chirp is instead a sawtooth shape that is reconverted to spatial modulation by the chicane. Fig. 4 shows high charge and low charge time profiles taken under identical conditions except for laser energy (UV light was attenuated). The variations in the time profile have been traced to the BBO laser harmonic generation crystals used to produce the UV light. It is found that the modulations are sensitive to the phase matching angle of these crystals. Investigations are underway to better understand this effect.

\section{COHERENT RADIATION MEASUREMENTS}

Fig. 5 shows coherent spectra for the three bunches of Fig. 3. The spectrum of the bunch at strong compression clearly exhibits a coherent signal at the microbunch frequency. These measurements and data from the coherent off-axis undulator experiment are detailed in companion papers [2,3]. There is strong coherent emission during microbunching. The strong compression case coupled $\sim 20 \mathrm{~nJ}$ or $\sim 3 \mathrm{E} 13$ IR photons into the detector.

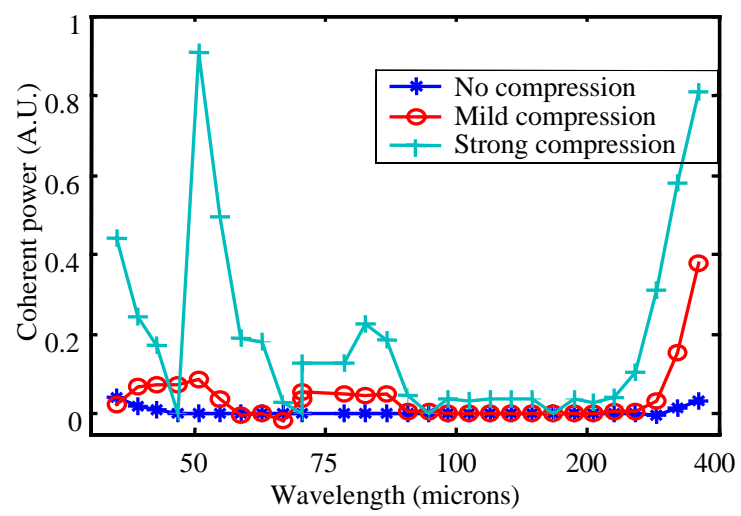

Figure 5: Coherent radiation spectra for same 3 compression measurements shown in Fig. 3.

\section{STREAK CAMERA MEASUREMENTS}

A Hamamatsu FESCA 500 sub-ps streak camera is installed and is used to measure laser time profiles. This camera has reflective input optics that can couple wavelengths from $200-1600 \mathrm{~nm}$, a broad response cathode sensitive from $200-900 \mathrm{~nm}$, optical trigger with less than $500 \mathrm{fs}$ jitter, full window time ranges from $50 \mathrm{ps}-6 \mathrm{~ns}$, and synchroscan unit to phase lock to external timing signals.

We have measured the FWHM time resolution to be 765 fs for repetitive sampling of the $800 \mathrm{~nm}$ Ti:Sapp oscillator, $1.01 \mathrm{ps}$ for single-shot measurements of the $800 \mathrm{~nm}$ Ti:Sapp amplifier, and $2.4 \mathrm{ps}$ for single-shot measurements of the $266 \mathrm{~nm}$ UV light. Single-shot measurements are degraded by space charge in the camera. The UV time response is further degraded by the energetic MCP electrons produced by hard photons.
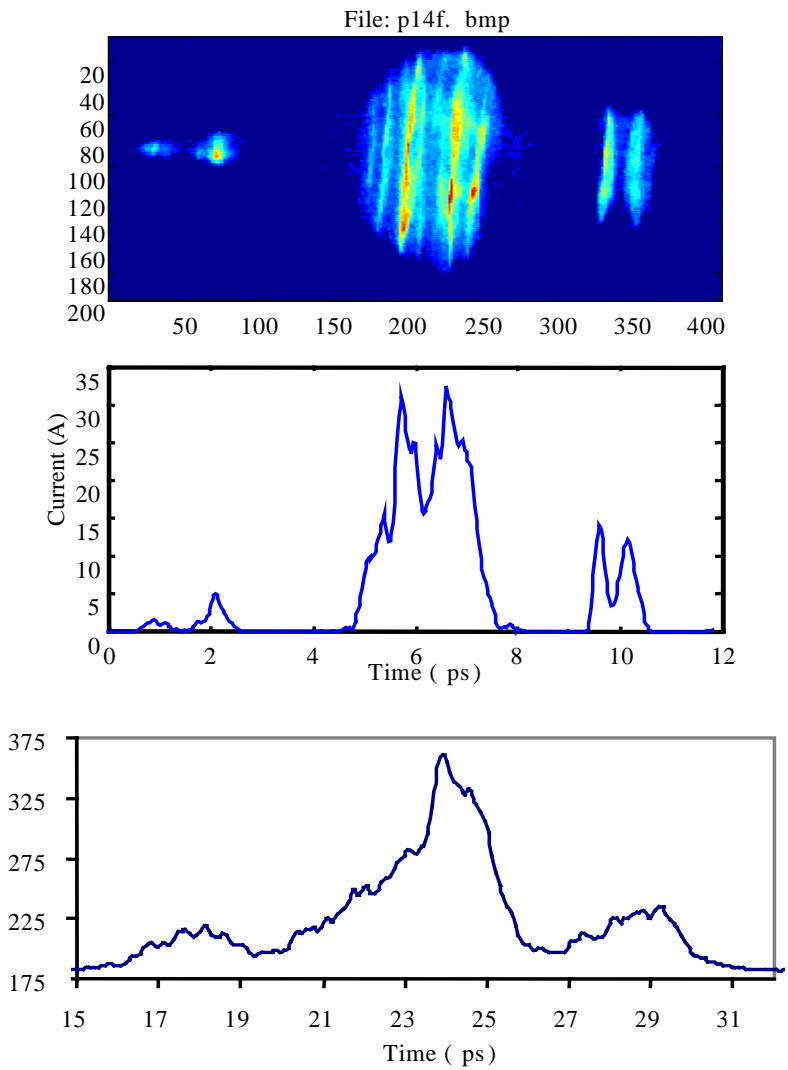

Figure 6: TOP: RF zero phasing image. MIDDLE: RF zero phasing time profile. BOTTOM: Streak profile of UV laser pulse.

Fig. 6 shows a comparison of a streak image of the UV laser pulse with RF-zero phasing electron pulse length measurements. In these images, the laser harmonic generation crystals were deliberately mistuned to produce satellites of the main pulse separated by a few ps. The main satellites are visible in the streak image, but the fine detail is hidden. The extraordinary time resolution of the RF zero phasing method resolves the sub-ps structure of the pulse.

\section{REFERENCES}

[1] D.X. Wang and G.A. Krafft, in Proceedings of the 1997 Particle Accelerator Conference (Vancouver, B.C., 1997), p. 2020.

[2] G.L. Carr et al, "Coherent Radiation Measurements at the NSLS Source Development Lab", in these proceedings (Chicago, IL, 2001).

[3] C.P. Neuman et al, "Measurement of Coherent OffAxis Undulator Radiation as a Beam Diagnostic", in these proceedings (Chicago, IL, 2001). 